\title{
X-ray flare in XRF 050406: evidence for prolonged engine activity
}

\author{
P. Romano ${ }^{1}$, A. Moretti ${ }^{1}$, P. L. Banat ${ }^{1}$, D. N. Burrows ${ }^{2}$, S. Campana ${ }^{1}$, G. Chincarini ${ }^{1}$, S. Covino ${ }^{1}$, D. Malesani ${ }^{3}$, \\ G. Tagliaferri ${ }^{1}$, S. Kobayashi ${ }^{2,4}$, B. Zhang ${ }^{5}$, A. D. Falcone ${ }^{2}$, L. Angelini ${ }^{6,7}$, S. Barthelmy ${ }^{6}$, A. P. Beardmore ${ }^{8}$, \\ M. Capalbi ${ }^{9}$, G. Cusumano ${ }^{10}$, P. Giommi ${ }^{11}$, M. R. Goad ${ }^{8}$, O. Godet ${ }^{8}$, D. Grupe ${ }^{2}$, J. E. Hill ${ }^{6,12}$, J. A. Kennea ${ }^{2}$, \\ V. La Parola ${ }^{10}$, V. Mangano ${ }^{10}$, P. Mészáros ${ }^{2}$, D. C. Morris ${ }^{2}$, J. A. Nousek ${ }^{2}$, P. T. O’Brien ${ }^{8}$, J. P. Osborne ${ }^{8}$,

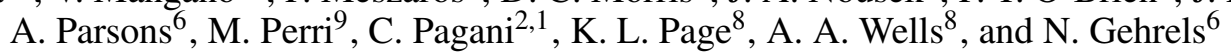

1 INAF - Osservatorio Astronomico di Brera, via E. Bianchi 46, 23807 Merate (LC), Italy e-mail: romano@merate.mi .astro.it

2 Department of Astronomy \& Astrophysics, Pennsylvania State University, 525 Davey Lab, University Park, PA 16802, USA

3 International School for Advanced Studies (SISSA-ISAS), via Beirut 2-4, 34014 Trieste, Italy

${ }^{4}$ Center for Gravitational Wave Physics, Pennsylvania State University, 104 Davey Lab, University Park, PA 16802, USA

5 Department of Physics, University of Nevada, Las Vegas, NV 89154-4002, USA

${ }^{6}$ NASA/Goddard Space Flight Center, Greenbelt, MD 20771, USA

7 Department of Physics and Astronomy, The Johns Hopkins University, 3400 North Charles Street, Baltimore MD 21218 USA

8 X-Ray and Observational Astronomy Group, Department of Physics \& Astronomy, University of Leicester, LE1 7RH, UK

9 ASI Science Data Center, via G. Galilei, 00044 Frascati, Roma, Italy

10 INAF - Istituto di Astrofisica Spaziale e Fisica Cosmica Sezione di Palermo, via U. La Malfa 153, 90146 Palermo, Italy

11 Agenzia Spaziale Italiana, Unità Osservazione dell’Universo, Viale Liegi 26, 00198 Roma, Italy

12 Universities Space Research Association, 10211 Wincopin Circle, Suite 500, Columbia, MD, 21044-3432, USA

Received 7 September 2005 / Accepted 3 January 2006

\section{ABSTRACT}

We present observations of XRF 050406, the first burst detected by Swift showing a flare in its X-ray light curve. During this flare, which peaks at $t_{\text {peak }} \sim 210 \mathrm{~s}$ after the BAT trigger, a flux variation of $\delta F / F \sim 6$ in a very short time $\delta t / t_{\text {peak }} \ll 1$ was observed. Its measured fluence in the $0.2-10 \mathrm{keV}$ band was $\sim 1.4 \times 10^{-8} \mathrm{erg} \mathrm{cm}^{-2}$, which corresponds to $1-15 \%$ of the prompt fluence. We present indications of spectral variations during the flare. We argue that the producing mechanism is late internal shocks, which implies that the central engine is still active at $210 \mathrm{~s}$, though with a reduced power with respect to the prompt emission. The X-ray light curve flattens to a very shallow slope with decay index of $\sim 0.5$ after $\sim 4400 \mathrm{~s}$, which also supports continued central engine activity at late times. This burst is classified as an X-ray flash, with a relatively low fluence $\left(\sim 10^{-7} \mathrm{erg} \mathrm{cm}^{-2}\right.$ in the $15-350 \mathrm{keV}$ band, $E_{\mathrm{iso}} \sim 10^{51} \mathrm{erg}$ ), a soft spectrum (photon index 2.65), no significant flux above $\sim 50 \mathrm{keV}$ and a peak energy $E_{\mathrm{p}}<15 \mathrm{keV}$. XRF 050406 is one of the first examples of a well-studied X-ray light curve of an XRF. We show that the main afterglow characteristics are qualitatively similar to those of normal GRBs. In particular, X-ray flares superimposed on a power-law light curve have now been seen in both XRFs and GRBs. This indicates that a similar mechanism may be at work for both kinds of events.

Key words. gamma rays: bursts - X-rays: bursts - X-rays: individuals: XRF 050406

\section{Introduction}

The Swift Gamma-Ray Burst Explorer (Gehrels et al. 2004) was successfully launched on 2004 Nov. 20. Its payload includes one wide-field instrument, the gamma-ray (15-350 keV) Burst Alert Telescope (BAT; Barthelmy et al. 2005a), and two narrow-field instruments, the X-Ray Telescope (XRT; Burrows et al. 2005a) and the Ultraviolet/Optical Telescope (UVOT; Roming et al. 2005). BAT detects the bursts, calculates their position to $\sim 1-4^{\prime}$ accuracy and triggers an autonomous slew of the observatory to point the two narrowfield instruments. The XRT, which operates in the $0.2-10 \mathrm{keV}$ energy range, can provide $\sim 5^{\prime \prime}$ positions, while the UVOT, which operates in the 1700-6000 $\AA$ wavelength range, can further refine the afterglow localization to $\sim 00^{\prime} 5$. With its unique fast re-pointing capabilities Swift set out to investigate the very early phases of gamma-ray burst (GRB) afterglows, beginning as early as one minute after the BAT trigger. During the initial activation and calibration phases, which ended on 2005 Apr. 5, Swift discovered 25 GRBs. The narrow-field instruments were re-pointed towards seven of them within a few hundred seconds, and such is the case for GRB 050406.

On 2005 Apr. 6 at 15:58:48.40 UT, BAT triggered on GRB 050406 (trigger 113872; Parsons et al. 2005), and located 
it at $\mathrm{RA}(\mathrm{J} 2000)=02^{\mathrm{h}} 17^{\mathrm{m}} 53^{\mathrm{s}}, \operatorname{Dec}(\mathrm{J} 2000)=-50^{\circ} 10^{\prime} 52^{\prime \prime}$, with an uncertainty of 3 arcmin (95\% containment; Krimm et al. 2005). The derived value for the time during which $90 \%$ of the burst fluence is observed was $T_{90}=5 \pm 1 \mathrm{~s}$ in the $15-350 \mathrm{keV}$ band. In the 15-25 keV band the light curve peak had a fastrise, exponential decay (FRED) profile, while in the $25-50 \mathrm{keV}$ band, the shape was more symmetric, with the peak starting $\sim 2 \mathrm{~s}$ earlier (Krimm et al. 2005). Both the peak and timeaveraged spectra were well fit by a simple power-law with a time-averaged spectrum photon index of $2.38 \pm 0.34$ ( $90 \%$ confidence; Krimm et al. 2005). The fluence in the $15-350 \mathrm{keV}$ band was $9.0 \times 10^{-8} \mathrm{erg} \mathrm{cm}^{-2}$. The gamma-ray characteristics of this burst, i.e. the softness of the observed spectrum and the absence of significant emission above $\sim 50 \mathrm{keV}$, classify GRB 050406 as an X-ray flash (XRF; Heise et al. 2001). From now on, we shall therefore refer to this event as XRF 050406.

Swift executed a prompt slew. The XRT imaged the BAT field only $84 \mathrm{~s}$ after the trigger but no bright X-ray source could be detected within the field of view. However, a refined on-ground analysis revealed a previously uncatalogued X-ray source (Cusumano et al. 2005a; Capalbi et al. 2005). From the very first examination of the down-linked data it was clear that the afterglow of this burst was peculiar. Indeed, after an initial decay, the X-ray count rate began rising, peaking at $\approx 220$ s, and subsequently decaying again (Capalbi et al. 2005).

Ground-based observations started as soon as the burst discovery was reported via the GCN network. The Magellan/Clay telescope imaged the XRT error circle with LDSS-3 in the $R$ and $i$ bands and found a single faint source $(R=22.0 \pm$ $0.09 \mathrm{mag}, 7.8 \mathrm{~h}$ after the burst) located at $\mathrm{RA}(\mathrm{J} 2000)=$ $02^{\mathrm{h}} 17^{\mathrm{m}} 52^{\mathrm{s}} .3, \operatorname{Dec}(\mathrm{J} 2000)=-50^{\circ} 11^{\prime} 15^{\prime \prime}$ with an uncertainty of $\sim 0.5$ in each coordinate (Berger et al. 2005a,b). Similarly to XRT, UVOT also imaged the field at the end of the slew (starting from $\sim 88 \mathrm{~s}$ after the trigger) and though it failed to detect the afterglow on-board (Landsman et al. 2005), subsequent on-ground analysis revealed a source within the XRT error circle at the 4.3- (19.0 mag), 3.0- and 2.5- $\sigma$ detection levels in the $U, B$ and $V$ bands, respectively. The UVOT position was $\mathrm{RA}(\mathrm{J} 2000)=02^{\mathrm{h}} 17^{\mathrm{m}} 52^{\mathrm{s}} .2, \operatorname{Dec}(\mathrm{J} 2000)=-50^{\circ} 11^{\prime} 15^{\prime \prime} .8$, consistent with the Magellan one. By the time the second UVOT observation ( $1.3 \mathrm{~h}$ later) was performed, the source was not detected in the $U$ band, confirming it as the afterglow of XRF 050406. Schady et al. (2006) obtained an estimate of $z=2.44 \pm 0.36$ from fitting the broad band spectrum (combined UVOT and XRT data).

In this paper we present observations of the first Swift burst where a flare is clearly detected in its X-ray light curve, during which the source count rate increased by a factor of $\gtrsim 6$. This feature had never been observed before in Swift data, and had rarely been observed before in any X-ray afterglow (Piro et al. 2005). This paper is organized as follows. In Sect. 2 we describe our observations and data reduction; in Sect. 3 we describe our spatial, timing and spectral data analysis; in Sect. 4 we discuss our findings. Finally, in Sect. 5 we summarize our conclusions. Throughout this paper the quoted uncertainties are given at $90 \%$ confidence level for one interesting parameter (i.e., $\Delta \chi^{2}=2.71$ ) unless otherwise stated. Times are referred to the BAT trigger $T_{0}, t=T-T_{0}$. The decay and spectral indices are parameterized as follows, $F(v, t) \propto t^{-\alpha} v^{-\beta}$, where $F_{v}\left(\mathrm{erg} \mathrm{cm}^{-2} \mathrm{~s}^{-1} \mathrm{~Hz}^{-1}\right)$ is the monochromatic flux as a function of time $t$ and frequency $v$; we also use $\Gamma=\beta+1$ as the photon index, $N(E) \propto E^{-\Gamma}\left(\mathrm{ph} \mathrm{keV}^{-1} \mathrm{~cm}^{-2} \mathrm{~s}^{-1}\right)$.

\section{Observations and data reduction}

\subsection{BAT observations}

Table 1 reports the log of the observations that were used for this work. The BAT data were analyzed using the standard BAT analysis software distributed within FTOOLS v6.0. The burst is detected in the first two standard bands (15-25 and 25-50 keV) while virtually no flux is observed above $50 \mathrm{keV}$. We find $T_{90}=$ $6.1 \pm 1.0 \mathrm{~s}$ in the $15-150 \mathrm{keV}$ band.

The BAT spectra were extracted over the full time interval over which the burst was detected $\left(T_{\text {tot }}\right)$, in the interval covering the 1-s peak $T_{\text {peak }}$, and for the $T_{90}$ and $T_{50}$ intervals. Response matrices were generated with the task batdrmgen using the latest spectral redistribution matrices. For our spectral fitting (XSPEC v11.3.2) we considered the $15-150 \mathrm{keV}$ energy range. All spectra are well fit with a simple power law with photon index $\Gamma_{\gamma} \sim 2.65$ (see details in Table 2). There is no evidence of a spectral break within the BAT energy range, thus constraining the peak energy $E_{\mathrm{p}}<15 \mathrm{keV}$. The indices are steeper (softer) although consistent with the ones reported by Krimm et al. (2005), due to the different energy ranges used for the spectral fitting. No significant improvements are found using either a cutoff power-law or a Band model (Band et al. 1993). The 1-s peak photon flux was $\left(2.3_{-0.4}^{+2.8}\right) \times 10^{-8} \mathrm{erg} \mathrm{cm}^{-2} \mathrm{~s}^{-1}(15-350 \mathrm{keV}$ band), while the fluence was $\mathcal{F}=\left(1.0_{-0.36}^{+1.13}\right) \times 10^{-7} \mathrm{erg} \mathrm{cm}^{-2}(15-350 \mathrm{keV}$ band). This fluence corresponds to an isotropic-equivalent energy $E_{\text {iso }}=\left(1.4_{-0.6}^{+1.6}\right) \times 10^{51} \mathrm{erg}$ (in the rest frame $\left.52-1204 \mathrm{keV}\right)$ assuming $z=2.44 \pm 0.36$ (Schady et al. 2006).

\subsection{XRT observations}

In order to cover the dynamic range and rapid variability expected from GRB afterglows and to provide rapid-response, automated observations, XRT was designed to support different readout modes that optimize the collected information as the flux of the burst diminishes. The XRT supports four major readout modes, one imaging (IM), two timing, Piledup/Low-rate Photodiode (PuPD and LrPD) and Windowed Timing (WT), and one Photon-Counting (PC). A detailed description of XRT modes can be found in Hill et al. (2004). In the nominal operating state the mode switching is based on the source flux and is fully automated (auto state) to minimize pileup in the data.

The XRT observations of XRF 050406 started on 2005 Apr. 6 at 16:00:12 UT, only $84 \mathrm{~s}$ after the trigger, and ended on 2005 Apr. 22, thus summing up a total net exposure (in PC mode) of $\sim 163 \mathrm{ks}$ spread over a $\sim 16 \mathrm{~d}$ baseline. The monitoring is organized in 9 observations $(000,001,002,005,006,008,009,010,011)$ and 183 snapshots (continuous pointings at the target). This was the first burst to occur after the formal end of the calibration phase 
Table 1. Observation log of XRF 050406.

\begin{tabular}{|c|c|c|c|c|c|}
\hline Sequence & Obs/Mode & $\begin{array}{c}\text { Start time (UT) } \\
\text { (yyyy-mm-dd hh:mm:ss) }\end{array}$ & $\begin{array}{c}\text { End time (UT) } \\
\text { (yyyy-mm-dd hh:mm:ss) }\end{array}$ & $\begin{array}{c}\text { Exposure }^{a} \\
\text { (s) }\end{array}$ & $\begin{array}{c}\text { Time since trigger } \\
\text { (s) }\end{array}$ \\
\hline 00113872000 & BAT & 2005-04-06 15:53:48 & 2005-04-08 06:32:39 & $\ldots$ & -300 \\
\hline 00113872000 & XRT/IM & 2005-04-06 16:00:12 & 2005-04-06 16:00:14 & 2.5 & 84 \\
\hline 00113872000 & XRT/PuPD & 2005-04-06 16:00:17 & 2005-04-08 00:04:58 & 92 & 90 \\
\hline 00113872000 & XRT/LrPD & 2005-04-06 16:00:18 & 2005-04-08 09:33:32 & 681 & 91 \\
\hline 00113872000 & XRT/WT & 2005-04-06 16:00:20 & 2005-04-08 09:38:58 & 3728 & 92 \\
\hline 00113872000 & $\mathrm{XRT} / \mathrm{PC}$ & 2005-04-06 16:00:26 & 2005-04-08 09:49:03 & 49939 & 99 \\
\hline 00113872001 & $\mathrm{XRT} / \mathrm{PC}$ & 2005-04-08 09:49:39 & 2005-04-08 22:51:57 & 7431 & 150652 \\
\hline 00113872002 & $\mathrm{XRT} / \mathrm{PC}$ & 2005-04-09 00:14:14 & 2005-04-11 23:09:57 & 12558 & 202527 \\
\hline 00113872005 & $\mathrm{XRT} / \mathrm{PC}$ & 2005-04-12 00:36:42 & 2005-04-12 23:20:12 & 3912 & 463074 \\
\hline 00113872006 & $\mathrm{XRT} / \mathrm{PC}$ & 2005-04-13 00:29:18 & 2005-04-13 23:26:58 & 11291 & 549031 \\
\hline 00113872008 & $\mathrm{XRT} / \mathrm{PC}$ & 2005-04-16 00:48:33 & 2005-04-18 23:43:57 & 35937 & 809386 \\
\hline 00113872009 & $\mathrm{XRT} / \mathrm{PC}$ & 2005-04-20 01:13:27 & 2005-04-20 23:59:57 & 17937 & 1156479 \\
\hline 00113872010 & $\mathrm{XRT} / \mathrm{PC}$ & 2005-04-21 01:19:44 & $2005-04-21$ 23:59:58 & 12546 & 1243256 \\
\hline 00113872011 & XRT/PC & 2005-04-22 00:05:06 & $2005-04-22$ 22:48:57 & 11526 & 1325178 \\
\hline
\end{tabular}

${ }^{a}$ The exposure time is spread over several snapshots (single continuous pointing at the target) during each observation (with the exclusion of BAT and XRT/IM data).

Table 2. Spectral fit results.

\begin{tabular}{lllllll}
\hline \hline Spectrum & Photon index & $\begin{array}{l}N_{\mathrm{H}} \\
\left(10^{20} \mathrm{~cm}^{-2}\right)\end{array}$ & $\chi_{\text {red }}^{2}($ d.o.f.) & C-stat $(\%)^{a}$ & $\begin{array}{l}\text { Start time } \\
\left(\mathrm{s} \mathrm{since} T_{0}\right)\end{array}$ & $\begin{array}{l}\text { End time } \\
\left(\mathrm{s} \text { since } T_{0}\right)\end{array}$ \\
\hline BAT total & $2.63_{-0.36}^{+0.42}$ & $\ldots$ & $1.4(56)$ & $\ldots$ & -2.560 & 4.160 \\
BAT $T_{90}$ & $2.64_{-0.38}^{+0.46}$ & $\ldots$ & $1.3(56)$ & $\ldots$ & -2.432 & 3.648 \\
BAT $T_{50}$ & $2.65_{-0.48}^{+0.61}$ & $\ldots$ & $1.0(56)$ & $\ldots$ & -0.064 & 2.048 \\
BAT peak & $2.65_{-0.60}^{+0.82}$ & $\ldots$ & $0.9(56)$ & $\ldots$ & 0.064 & 1.024 \\
\hline XRT WT & $2.11_{-0.28}^{+0.31}$ & $2.8^{b}$ & $1.0(6)$ & $\ldots$ & 92 & 596 \\
XRT WT+PC & $2.12_{-0.23}^{+0.25}$ & $2.8^{b}$ & $1.0(8)$ & $\ldots$ & 92 & 599 \\
XRT PC & $2.13_{-0.19}^{+0.44}$ & $2.8^{b}$ & $\ldots$ & $220.7(58.0)$ & 99 & 599 \\
XRT PC & $2.06_{-0.24}^{+0.24}$ & $2.8^{b}$ & $\ldots$ & $270.7(67.1)$ & 599 & 18308 \\
\hline
\end{tabular}

${ }^{a}$ Cash statistic (C-stat) and percentage of Monte Carlo realizations that had statistic $<\mathrm{C}$-stat. We performed $10^{4}$ simulations.

${ }^{b}$ Fixed to the Galactic value.

${ }^{c}$ Snapshots 1 through 4 , with the exclusion of the first $\sim 600$ s (non piled-up data).

(2005 Apr. 5), and the first (000) observation was performed as an automated target (AT) with XRT in auto state. Therefore, during observation 000 the automated mode switching made XRT take an initial $2.5 \mathrm{~s}$ image (IM at $t=84 \mathrm{~s}$ ), immediately followed by one PuPD $(t=90 \mathrm{~s})$ and one $\operatorname{LrPD}(t=91 \mathrm{~s})$ frame. Then at $t=92 \mathrm{~s}$ a series of $5 \mathrm{WT}$ frames was taken until the on-board measured count rate was low enough for XRT to switch to PC mode $(t=99 \mathrm{~s})$. After this, XRT repeatedly switched between WT and PC modes because of an increased background level (see below). Since the signal-to-noise $(\mathrm{S} / \mathrm{N})$ in these late WT frames is low, we did not include them in our analysis (Table 1).

The XRT data were first processed by the Swift Data Center at NASA/GSFC into Level 1 products (event lists). Then they were further processed with the XRTDAS (v1.4.0) software package written by the Agenzia Spaziale Italiana (ASI) Science
Data Center and distributed within FTOOLS v6.0 to produce the final cleaned event lists. We ran the task xrtpipeline (v0.8.8) applying calibration and standard filtering and screening criteria, i.e., we cut out temporal intervals during which the CCD temperature was higher than $-47{ }^{\circ} \mathrm{C}$, and we removed hot and flickering pixels. These are present because the CCD is operating at a temperature higher than the design temperature of $-100{ }^{\circ} \mathrm{C}$ due to a failure in the active cooling system. An on-board event threshold of $\sim 0.2 \mathrm{keV}$ (un-reconstructed pulse-height PHAS[1] > 80) was also applied to the central pixel, which has been proven to reduce most of the background due to either the bright Earth limb or the CCD dark current (which depends on the CCD temperature). These two sources of background are the main reason for the switching between PC and WT mode even when the source count rate is below 1 counts $\mathrm{s}^{-1}$. 


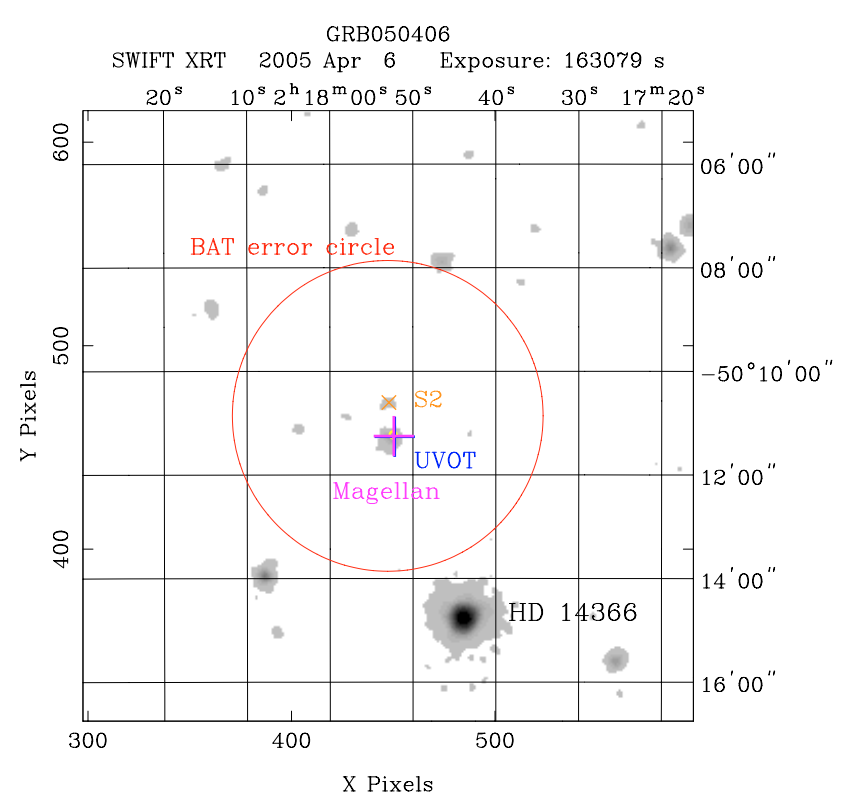

Fig. 1. XRT image of XRF 050406, obtained from the total $\sim 163 \mathrm{ks}$ PC mode data. The field is centred on the $3^{\prime}$ radius BAT error circle. Also shown is the XRT 4.'2 error circle, as well as the Magellan (Berger et al. 2005a) and UVOT (Rol et al. 2005) optical counterpart positions; the optical points are so close they cannot be distinguished on this scale. S2 is a serendipitous source located at RA(J2000) = $02^{\mathrm{h}} 17^{\mathrm{m}} 52^{\mathrm{s}} .9, \operatorname{Dec}(\mathrm{J} 2000)=-50^{\circ} 10^{\prime} 36^{\prime \prime} 1$.

Throughout the monitoring campaign the CCD temperature was $<-50{ }^{\circ} \mathrm{C}$, with the exception of part of observations 002 and 005 , where it became as high as -43.5 and $-45^{\circ} \mathrm{C}$, respectively; those data were therefore screened out. For our analysis we further selected XRT grades 0-12 and 0-2 for PC and WT data, respectively (according to Swift nomenclature; Burrows et al. 2005a).

\section{Data analysis}

\subsection{Spatial analysis}

Figure 1 shows the $163 \mathrm{ks}$ XRT image accumulated in PC mode in the $0.2-10 \mathrm{keV}$ energy band. We detected two previously uncatalogued sources within 1 arcmin of the optical burst coordinates. The brightest uncatalogued source, which we identified as the fading X-ray counterpart of the burst, is present in the first four XRT snapshots. The source is piled-up during the initial $500 \mathrm{~s}$ of PC data. Therefore, to obtain an unbiased position, we rely on the remainder of the PC data in the first observation, which has a net exposure of $49.8 \mathrm{ks}$. We used the xrtcentroid task (v0.2.7) and found that the afterglow position is $\mathrm{RA}(\mathrm{J} 2000)=2^{\mathrm{h}} 17^{\mathrm{m}} 52^{\mathrm{s}} .4, \operatorname{Dec}(\mathrm{J} 2000)=$ $-50^{\circ} 11^{\prime} 13^{\prime \prime}$. 6 . We estimate its uncertainty to be 4 .' 2 (90\% confidence level). This position takes into account the correction for the misalignment between the telescope and the satellite optical axis (Moretti et al. 2006). Figure 1 shows the XRT error circle, as well as the $3^{\prime}$ BAT error circle (Krimm et al. 2005; 95\% containment) and the optical counterpart coordinates determined by Magellan (Berger et al. 2005a) and by UVOT (Rol et al. 2005). The XRT coordinates are 23" from the BAT ones, and 1". 6 and 2.' 8 from the Magellan and UVOT ones, respectively. XRF 050406 was detected (XIMAGE v4.3) in the first four snapshots individually, but not from the 5th on. The second source, S2, is located at RA(J2000) $=02^{\mathrm{h}} 17^{\mathrm{m}} 52^{\mathrm{s}} .9$, $\operatorname{Dec}(\mathrm{J} 2000)=-50^{\circ} 10^{\prime} 36^{\prime \prime} .1$ and has a constant rate $(3.8 \pm 0.7) \times$ $10^{-4}$ counts $^{-1}$ throughout the observation campaign.

\subsection{Temporal analysis}

During the first $500 \mathrm{~s}$ of the XRT observation the intensity of the afterglow was high enough to cause pile-up in the PC mode data. To account for this effect we extracted the source events within an annulus with a 30-pixel outer radius $\left(\sim 71^{\prime \prime}\right)$ and a 2-pixel inner radius. These values were derived by comparing the observed and nominal PSF. For the PC data collected after the first $500 \mathrm{~s}$, the entire circular region (30-pixel radius) was used, instead. In both cases we further disregarded data within a circular region centred on the serendipitous source $\mathrm{S} 2$ (which lies within the 30-pixel PC source extraction region) with a 7.17 pixel $\left(17^{\prime \prime}\right)$ radius. The WT data were extracted in a rectangular region 31 pixels long along the image strip (and 20 pixels wide), which excludes the data from the source S2. The selected extraction regions correspond to $\sim 69 \%$ (piled-up PC), $\sim 95 \%$ (non piled-up PC), and $\sim 94 \%$ (WT) of the XRT PSF. To account for the background, data were also extracted in PC mode within a circular region (radius $130^{\prime \prime}=54.8$ pixels) and in WT mode within a rectangular box $(40 \times 20$ pixels $)$, in locations far from background sources. The mean PC background in the $0.2-10 \mathrm{keV}$ band was found to be constant throughout the observations and, normalized to the PC source extraction region, it had a value of $\sim 2.6 \times 10^{-3}$ counts s$^{-1}$. The mean WT background in the same energy band and normalized to the WT source region was $\sim 4.6 \times 10^{-2}$ counts s $^{-1}$.

Figure 2 shows the background-subtracted light curve extracted in the $0.2-10 \mathrm{keV}$ energy band, with the BAT trigger as origin of time. We considered WT data for the first snapshot of the first observation, and PC data for all 9 available observations (see Table 1). During the initial phases of the afterglow evolution $\left(t<4 \times 10^{4} \mathrm{~s}\right)$ we binned the source counts with a minimum of 30 counts per time bin, and dynamically subtracted the normalized background counts in each bin. The PC mode data were corrected for the effects of pile-up. We note that, by keeping to the minimum number of counts per time bin criterion, we created several bins during the first snapshot, but subsequently needed to merge data belonging to snapshots 1 and 2 (point at $\sim 4 \mathrm{ks}$ ), then from snapshots 3 and 4 (point at $\sim 20 \mathrm{ks}$ ), and later on from snapshots 5 through 8 (point at $\sim 35 \mathrm{ks}$ ). Afterwards, we used XIMAGE with the option SOSTA, which calculates vignetting- and PSF-corrected count rates within a specified box, and the background in a user-specified region. To ensure uniformity with the early light curve, the background was estimated in the same region as the one used for the initial part of the light curve. We thus obtained a signal-to-noise ratio $S / N \gtrsim 3$ (the only exception being the point at $\sim 33 \mathrm{ks}$ which has $S / N \gtrsim 2$ ). The last point is a 3- $\sigma$ upper limit. This latter method is preferred for the construction of the late part of the light curve since it better accounts for 


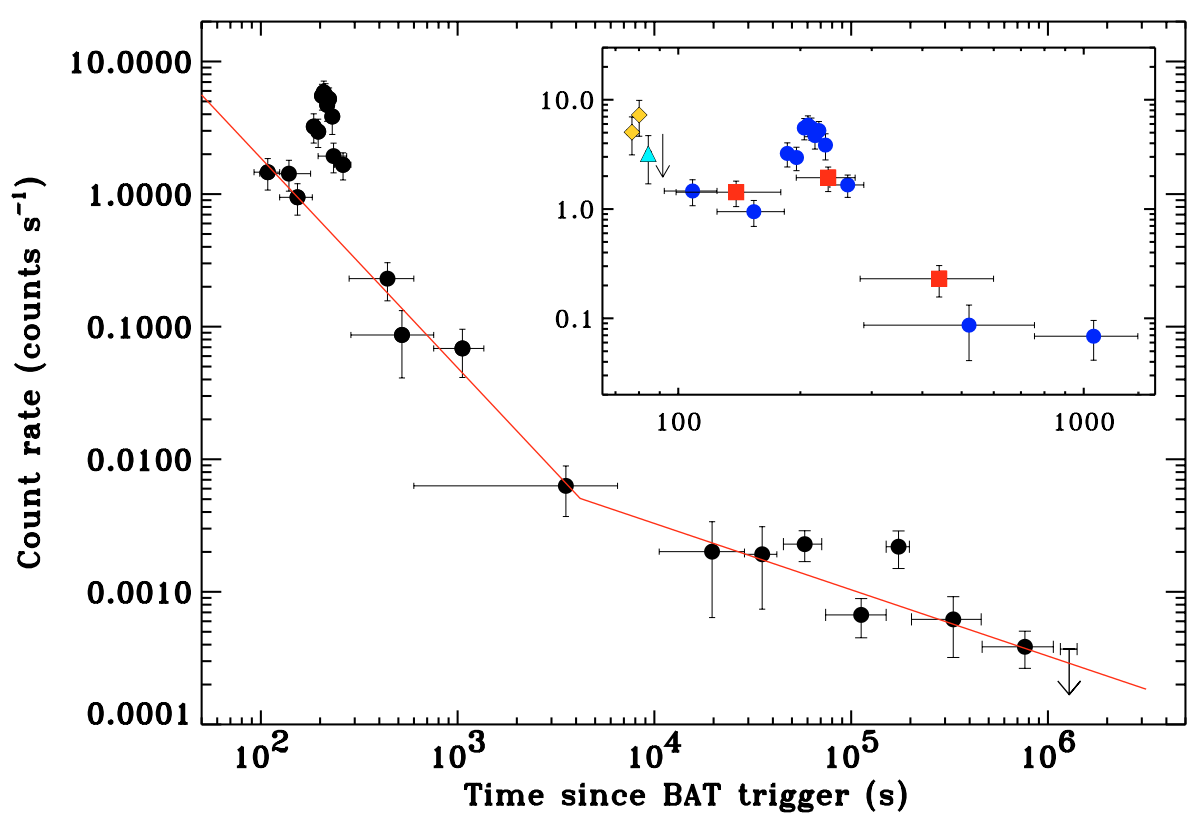

Fig. 2. X-ray light curve of the XRF 050406 afterglow in the $0.2-10 \mathrm{keV}$ energy band. The curve is background-subtracted and the time is referred to the BAT trigger, 2005 Apr. 06 at 15:58:48.4 UT (Parsons et al. 2005). The last point after $10^{6} \mathrm{~s}$ is a 3- $\sigma$ upper limit. Inset: details of the first $\sim 1000 \mathrm{~s}$, which include data in all XRT modes. The (yellow) diamonds represent LrPD mode data taken during the latter portion of the slewing phase; the (cyan) triangle is the initial IM point ( $84 \mathrm{~s}$ after the trigger, see Table 1), the downward-pointing arrow is a LrPD limit (pointing, $91 \mathrm{~s}$ after the trigger), the (blue) circles are WT mode data (starting from $92 \mathrm{~s}$ after the trigger), and the (red) squares are PC mode data (starting from $99 \mathrm{~s}$ after the trigger). The data have been corrected for pile-up (where appropriate) and PSF losses. The solid (red) line represents the best-fit broken powerlaw model to the light curve (excluding the flare).

Table 3. Light curve fit results ${ }^{a}$.

\begin{tabular}{lllll}
\hline \hline Model parameters & $\begin{array}{l}\text { Simple power law } \\
\text { excluding flare }\end{array}$ & $\begin{array}{l}\text { Broken power law } \\
\text { excluding flare }\end{array}$ & $\begin{array}{l}\text { Smoothly-joined power laws } \\
\text { excluding flare }\end{array}$ & $\begin{array}{l}\text { Broken power law+Gaussian } \\
\text { full data set }\end{array}$ \\
\hline$\alpha_{1}$ & $1.41_{-0.24}^{+0.22}$ & $1.58_{-0.16}^{+0.18}$ & $1.73_{-0.24}^{+0.40}$ & $1.58 \pm 0.17$ \\
$t_{\mathrm{b}}(\mathrm{s})$ & - & $\left(4.19_{-0.36}^{+6.17}\right) \times 10^{3}$ & $\left(3.61_{-1.03}^{+1.36}\right) \times 10^{3}$ & $\left(4.36_{-0.53}^{+6.23}\right) \times 10^{3}$ \\
$\alpha_{2}$ & - & $0.50 \pm 0.14$ & $0.42_{-0.12}^{+0.11}$ & $0.50_{-0.14}^{+0.13}$ \\
Gaussian centre (s) & - & - & - & $211.1_{-4.4}^{+5.4}$ \\
Gaussian width (s) & - & - & - & $17.9_{-4.6}^{+12.3}$ \\
$\chi_{\text {red }}^{2}$ & 4.32 & 1.20 & 1.29 & 1.58 \\
d.o.f. & 12 & 10 & 10 & 17 \\
\hline
\end{tabular}

\footnotetext{
${ }^{a}$ We follow the notation $F(t) \propto t^{-\alpha}$.
}

the background in a low-counts regime. We note, however, that extracting the light curve in the same 30-pixel source region up to the end of the last observation, we obtained fully consistent results, albeit with a noisier light curve. We also note that the residual contribution of the serendipitous source S2 within the source extraction region is $\$ 19 \%$ of the S2 counts, which corresponds to $\lesssim(7 \pm 1) \times 10^{-5}$ counts s$^{-1}$. Therefore, S2 only makes a marginal contribution to the afterglow light curve, which amounts to $<20 \%$ of the last point.

The light curve clearly shows a complex behaviour, with a power law decay underlying a remarkable flare which peaks at $\approx 210 \mathrm{~s}$ after the BAT trigger (see Fig. 2, inset). To fit the light curve we used the BAT trigger as reference time and we only considered spectroscopic-mode data obtained while XRT was pointing, thus excluding the early LrPD, the LrPD upper limit and the IM point. Further excluding the data taken during the flare $(180 \mathrm{~s}<t<300 \mathrm{~s})$, a fit with a simple power law yields $\chi_{\text {red }}^{2}=4.32$ (12 degrees of freedom, d.o.f.), which is unacceptable. A fit with a broken power law $F(t)=K t^{-\alpha_{1}}$ for $t<t_{\mathrm{b}}$ and $F(t)=K t_{\mathrm{b}}^{-\alpha_{1}}\left(t / t_{\mathrm{b}}\right)^{-\alpha_{2}}$ for $t>t_{\mathrm{b}}$, where $t_{\mathrm{b}}$ is the time of the break, yields $\alpha_{1}=1.58_{-0.16}^{+0.18}$ and $\alpha_{2}=0.50 \pm 0.14$, and a break at $\sim 4200 \mathrm{~s}$ after the BAT trigger. This latter model yields a good fit $\left(\chi_{\text {red }}^{2}=1.20,10\right.$ d.o.f. $)$, a significant improvement over the simple power law (null hypothesis probability $=1.7 \times 10^{-3}$, equivalent to $3.2 \sigma$ ), but some of the parameters are not well constrained. Alternatively, a fit with two smoothly joined power laws $F(t)=K^{\prime}\left[\left(t / t_{\mathrm{b}}\right)^{-\alpha_{1}}+\left(t / t_{\mathrm{b}}\right)^{-\alpha_{2}}\right]$ yields $\chi_{\text {red }}^{2}=1.29$ (10 d.o.f.) with similar values for the inferred parameters. A summary of the fits to the light curve can be found in Table 3. As a reference, the $0.2-10 \mathrm{keV}$ unabsorbed flux at $t_{\mathrm{b}}$ is $(4 \pm 1) \times 10^{-13} \mathrm{erg} \mathrm{cm}^{-2} \mathrm{~s}^{-1}$ (we adopted a count rate to unabsorbed flux conversion factor of $6.5 \times$ $10^{-11} \mathrm{erg} \mathrm{cm}^{-2}$ count $^{-1}$, obtained from the best fit models derived in Sect. 3.3) and the luminosity in the $0.7-34.4 \mathrm{keV}$ band is $(1.9 \pm 0.9) \times 10^{46} \mathrm{erg} \mathrm{s}^{-1}$.

During the flare a rebrightening of the source by a factor of $\gtrsim 6$ in flux was observed between $t \sim 154 \mathrm{~s}$ and the peak at $\sim 210 \mathrm{~s}$. Both the rising and the falling part of the flare had very steep slopes that, when fit with a simple power law, yield $\alpha_{1, \text { flare }}=-5.8_{-2.1}^{+1.6}$ and $\alpha_{2, \text { flare }}=6.7 \pm 1.0$. When the underlying power-law afterglow is subtracted, the fit yields $\alpha_{1, \text { flare }}=$ $-6.8_{-2.1}^{+2.4}$ and $\alpha_{2, \text { flare }}=6.8_{-2.0}^{+3.6}$ and the peak is at $213 \pm 7 \mathrm{~s}$ from the BAT trigger. In all cases the errors are dominated by the uncertainty in the placement of the flare boundaries. The 


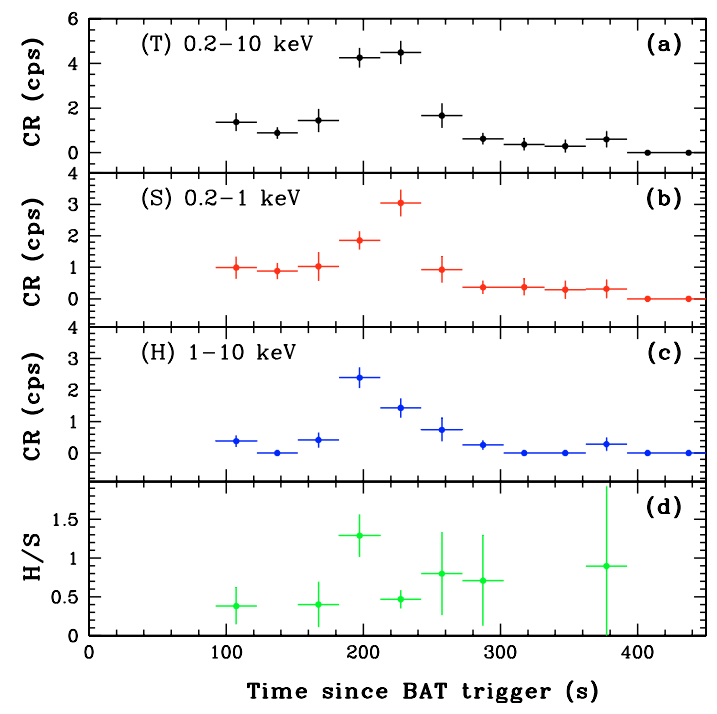

Fig. 3. WT background-subtracted light curves. a): Total band (T, $0.2-10 \mathrm{keV})$. b): Soft band $(\mathbf{S}, 0.2-1 \mathrm{keV})$. c): Hard band $(\mathbf{H}$, 1-10 keV). d): Ratio of hard to soft count rates.

flare can also be characterised, as a simple parametric description, as a Gaussian line. A combined broken power law and Gaussian model fit yields a peak at $211.1_{-4.4}^{+5.4} \mathrm{~s}\left(61.4_{-1.3}^{+1.6} \mathrm{~s}\right.$ in the rest frame) and a width $17.9_{-4.6}^{+12.3} \mathrm{~s}\left(\chi_{\text {red }}^{2}=1.58,17\right.$ d.o.f. $)$. In this case the ratio of the characteristic time-scale and the peak time is $\delta t / t_{\text {peak }} \sim 0.08$ or 0.20 , when using the Gaussian width or its FWHM $\left(42.2_{-10.8}^{+29.0} \mathrm{~s}\right)$, respectively. In either case, $\delta t / t_{\text {peak }} \ll 1$, which puts severe constraints on the emission mechanisms that can produce the flare. We shall address this issue in the discussion section. Integration of the Gaussian bestfitting function yields an estimate of the fluence of the flare, $(1.4 \pm 1.0) \times 10^{-8} \mathrm{erg} \mathrm{cm}^{-2}$, corresponding to an energy of $(2.0 \pm 1.4) \times 10^{50} \mathrm{erg}$. The large error reflects the uncertainty on the actual model used for the integration of the flare.

We also extracted events from the first snapshot WT data in two more energy bands, $0.2-1 \mathrm{keV}$ (soft, S) and 1-10 keV (hard, $\mathrm{H}$ ), as well as the total band, $0.2-10 \mathrm{keV}$. We used the same regions as the ones described above, a constant time binning of $30 \mathrm{~s}$ and dynamically subtracted their respective backgrounds. Figure 3 shows the three background-subtracted light curves, as well as the ratio H/S. Indeed, during the rising portion of the flare the hard band flux increases by a factor of $\gtrsim 6$ while the soft band flux only increases slightly, so that the spectrum of the flare starts off harder than the underlying afterglow, and then evolves into a softer state as its flux decreases; this can be seen in the following time bin, when the soft band flux peaks with a flare to pre-flare flux ratio of $\sim 3.5$. This yields an indication of spectral evolution during the flare as a $\sim 3-\sigma$ excess over a constant fit to $\mathrm{H} / \mathrm{S}$. It should be noted that this behaviour is reminiscent of that observed in the prompt emission (Ford et al. 1995), with the harder band peak preceding the softer band peak.

At $t \sim 1.7 \times 10^{5} \mathrm{~s}$ a second faint bump is observed. Its significance is not high, since it is detected as a $2-\sigma$ excess over the underlying afterglow. Similar late-time bumps have been observed in other Swift-detected GRBs (e.g. GRB 050502B; Falcone et al. 2006).

\subsection{Spectral analysis}

The afterglow of XRF 050406 was very faint, hence it is not possible to perform time-resolved spectroscopy to distinguish the spectral properties of the afterglow proper from the ones of the flare observed in the light curve. Therefore, we proceeded as follows. Spectra of the source and background were extracted in the regions described in Sect. 3.2 from the first observation (000) event files. PC and WT spectra were extracted during the first $\sim 500 \mathrm{~s}$ of the PC observation (see Table 2 for times referred to $T_{0}$ ), when PC data are piled-up and when the flare is observed in the light curve. We also extracted PC spectra after the first $500 \mathrm{~s}$ during the first 4 snapshots. For the latter we used a circular region with a 10 pixel radius (corresponding to $\sim 80 \%$ of the XRT PSF) to minimize the background and to be able to use the Cash statistics (Cash 1979). Ancillary response files (ARF) were generated with the task xrtmkarf within FTOOLS v6.0 using the latest ARF distribution (v003). These ARFs account for different extraction regions and PSF corrections. We used the latest spectral redistribution matrices (v007). The energy ranges adopted for spectral fitting were $0.5-10 \mathrm{keV}$ and $0.2-10 \mathrm{keV}$ for WT and PC, respectively.

We first performed a fit with an absorbed (wabs in XSPEC) power law to the WT data (166 counts), which were rebinned with a minimum of 20 counts per energy bin to allow $\chi^{2}$ fitting within XSPEC. The hydrogen column was initially kept as a free parameter, and then frozen to the Galactic value $\left(N_{\mathrm{H}}^{\mathrm{G}}=2.8 \times 10^{20} \mathrm{~cm}^{-2}\right.$, Dickey \& Lockman 1990) when the fit yielded a value lower than (although consistent with) the Galactic one. The fit was good, $\chi_{\text {red }}^{2}=1.0$ for 6 d.o.f., and yielded $\Gamma=2.11_{-0.28}^{+0.31}$. We then performed a fit with the same model to the remainder of the PC data during snapshots 1 through 4 using Cash statistics which is more appropriate given the low number of counts (21 un-binned counts) and calculated the goodness of the fit via $10^{4}$ Montecarlo simulations. The fit was good and yielded consistent results. We also performed simultaneous fits to the WT and PC (60 counts) spectra extracted during the first $\sim 500 \mathrm{~s}$ (using $\chi^{2}$ statistics) and of the PC data alone (using Cash statistics), also obtaining consistent results. Table 2 summarizes the results of the fits. We note that, given the current goodness of the XRT calibration (5\% systematic uncertainty for all observing modes and grade selections in the 0.5-10 keV range; e.g., Romano et al. 2005), an excess of $N_{\mathrm{H}}$ cannot be excluded and we find a 3- $\sigma$ upper limit to the total (Galactic plus intrinsic) hydrogen column along the line of sight of $N_{\mathrm{H}}<9 \times 10^{20} \mathrm{~cm}^{-2}$.

We can therefore conclude that, during the first $600 \mathrm{~s}$ after the burst, which include the X-ray flare observed in the light curve, the mean photon index is $\Gamma=2.1 \pm 0.3$, and that the photon index does not vary after the end of the flare. However, we do have clues regarding the presence of spectral evolution during the flare coming from the hardness ratio analysis (Sect. 3.2), even though the statistics are not high enough to show it in the spectral analysis. As we will discuss later (Sect. 4.2), other 
afterglows with larger amplitude X-ray flares demonstrate a strong spectral evolution of the flares.

\section{Discussion}

\subsection{Gamma-ray properties: similarity of XRFs and GRBs}

The duration of this burst $\left(T_{90}=5 \pm 1 \mathrm{~s}\right.$ in the $15-350 \mathrm{keV}$ band) places this burst in the short tail of the long GRB population (Kouveliotou et al. 1993). Its fluence is relatively low $\left(1.0 \times 10^{-7} \mathrm{erg} \mathrm{cm}^{-2}\right.$ in the $15-350 \mathrm{keV}$ band) but not unusually faint. The gamma-ray characteristics of this burst are consistent with a classification as an X-ray flash (Heise et al. 2001), or as an "X-ray rich GRB" (XRR). The softness of the observed spectrum, which is well fit in the $15-150 \mathrm{keV}$ band with a simple power law with photon index $\Gamma_{\gamma}=2.65$, and with no significant emission observed above $\sim 50 \mathrm{keV}$, implies that the peak energy is below the BAT bandpass $\left(E_{\mathrm{p}}<15 \mathrm{keV}\right)$. The operational definition of XRFs/XRRs (e.g. Lamb et al. 2004) is of a fast transient $\mathrm{X}$-ray source characterized by a softness ratio $\mathrm{SR}=\log [\mathcal{F}(2-30 \mathrm{keV}) / \mathcal{F}(30-400 \mathrm{keV})]>0$ for an XRF and $-0.5<\mathrm{SR}<0$ for an XRR. Extrapolation of the BAT spectrum, with the assumption of $E_{\mathrm{p}}<2 \mathrm{keV}$, yields $\mathrm{SR}=0.8_{-0.4}^{+0.5}$, which classifies this burst as an XRF. However, a break in the spectrum may well be present in the $2-15 \mathrm{keV}$ band. In the most conservative case, i.e. assuming no flux below $15 \mathrm{keV}$, this event would be an XRR GRB, with SR $=-0.2_{-0.3}^{+0.2}$.

The isotropic-equivalent gamma-ray energy of this event is $E_{\text {iso }}=\left(1.4_{-0.6}^{+1.6}\right) \times 10^{51} \mathrm{erg}$ (Sect. 2.1), and this effectively puts XRF 050406 in the low-energy tail of GRB energies (Bloom et al. 2003b). Assuming that the Amati relation (Amati et al. 2002) holds, we can infer a rest-frame $E_{\mathrm{p}}^{\text {rest }} \sim 55 \mathrm{keV}$, which corresponds to an observer-frame $E_{\mathrm{p}} \sim 15 \mathrm{keV}$. This value is consistent with the nondetection of $E_{\mathrm{p}}$ in the BAT energy range.

To date, X-ray afterglows of XRFs have been detected in just a few cases (XRF 011030, XRF 020427: Bloom et al. 2003a; Levan et al. 2005; XRF 030723: Butler et al. 2004; XRF 040701: Fox 2004; XRF 050315: Vaughan et al. 2006). This is one of the first examples of a well-studied X-ray light curve of an XRF. Its main characteristics are not qualitatively different from those of normal GRBs (Chincarini et al. 2005; Nousek et al. 2006). As observations accumulate, it is becoming clear that these two classes of phenomena share many properties, and both have afterglows with similar characteristics (Sakamoto et al. 2005). This is a clue that both types of events may have a common origin and is supported by recent evidence that some XRFs are associated with supernovae (Soderberg et al. 2004; Bersier et al. 2005; Fynbo et al. 2004).

\subsection{X-ray flares: evidence for prolonged engine activity}

The general behaviour of the afterglow of XRF 050406 is a typical one. The observed X-ray photon index $\left(\Gamma_{X}=2.1\right)$ is common among X-ray afterglows (Chincarini et al. 2005; De Pasquale et al. 2005). The light curve shows a break from a relatively steep decay $\left(\alpha_{1}=1.58\right)$ to a flatter one $\left(\alpha_{2}=0.50\right)$.
Its overall shape is similar to the one typically observed by the XRT (Chincarini et al. 2005; Nousek et al. 2006), even though the initial slope is less steep than average.

The most striking characteristic of this burst is the strong flare in its X-ray light curve, a feature which had never been detected by Swift before and had been previously observed in very few GRBs (GRB 970508, Piro et al. 1999; GRB 011121 and GRB 011211, Piro et al. 2005). The fluence of the flare is $\sim 1.4 \times 10^{-8} \mathrm{erg} \mathrm{cm}^{-2}$ in the $0.2-10 \mathrm{keV}$ band, which amounts to $\sim 14 \%$ of the observed (15-350 keV band) prompt fluence. A better estimate of the flare-to-prompt energy ratio would require the knowledge of the prompt spectral energy distribution (SED). Since the actual peak energy of the prompt SED is unknown $\left(E_{\mathrm{p}}<15 \mathrm{keV}\right)$, the extrapolation of the BAT fluence to the XRT band is highly uncertain. For plausible values of $E_{\mathrm{p}}$, the flare to prompt fluence ratio is in the $1-10 \%$ range. The observed rebrightening is by a factor of 6 in flux, presents a peak at $t_{\text {peak }}=213 \pm 7 \mathrm{~s}$ and takes place on a very short timescale, with a ratio of the characteristic time-scale and the peak time $\delta t / t_{\text {peak }} \ll 1$. Both the rising and the falling parts of the afterglow-subtracted flare had very steep slopes, $\alpha_{1, \text { flare }} \approx-7$ and $\alpha_{2 \text {,flare }} \approx 7$, assuming the burst trigger as the time origin.

According to the standard relativistic fireball model, the prompt emission is caused by internal shocks within the expanding fireball, while the afterglow is produced by the fireball shocking the external medium (external shocks, Piran 1999; Zhang \& Mészáros 2004). Available models to explain flares include refreshed shocks (Rees \& Mészáros 1998), external shocks with a clumpy medium (Lazzati et al. 2002) and angular inhomogeneities in the outflow (Fenimore et al. 1999; Nakar et al. 2003). However, it can be argued (Burrows et al. 2005b, Zhang et al. 2005, Nousek et al. 2006) that such models cannot produce the observed large flux variations $\delta F / F_{\text {peak }} \gg 1$ in such short timescales $\delta t / t_{\text {peak }} \ll 1$ (Ioka et al. 2005). Similarly, none of the above mechanisms would explain the steep slopes observed in the flare. External reverse shocks, created when the fireball slows down because of the interaction with the external medium, are expected to emerge at optical and radio wavelengths, hence synchrotron self-Compton (SSC) must be invoked to produce emission in the X-ray band. This would require carefully balanced conditions (Kobayashi et al. 2005).

Piro et al. (2005) suggested that the X-ray flares observed in GRB 011121 and GRB 011211 were due to the onset of the afterglow. The steep slopes and the short timescale variability can only be accounted for within the thick shell scenario (Sari \& Piran 1999). Galli \& Piro (2005) successfully modeled XRF 011030 using this model. In this scenario, the emission before and after the flare is due to different processes (prompt tail and afterglow, respectively), hence a discontinuity in the light curve is generally expected underlying the flare. This is not the case for XRF 050406, where the same component describes the X-ray emission both before and after the flare. Even if a fine-tuning may explain this particular event, the lack of a light curve break is common to a large fraction of the flares observed by Swift (Burrows et al. 2005c). Therefore, while the explanation of flares in terms of the afterglow onset is 
attractive, it is unlikely to be applicable to the vast majority of the X-ray flares seen by XRT.

A promising mechanism to produce the flare is late internal shocks (Fan \& Wei 2005; Zhang et al. 2005; King et al. 2005; Perna et al. 2006), which implies that the central engine is still active at $t=213 \mathrm{~s}$, even though the prompt emission ended after $t \sim 6 \mathrm{~s}$. The late-time activity in this case must have a reduced power with respect to the prompt emission, as the relative fluences indicate. Such a mechanism would naturally explain the steep rise and decay slopes. Second, the energy required to power the flare would be much lower than in the other scenarios (Zhang et al. 2005). The indications of spectral evolution throughout the flare further support this interpretation. The flare appears to be harder than the underlying afterglow, which suggests a distinct origin for this emission. Furthermore, there are indications of spectral evolution, which shows the typical hard-to-soft pattern. Such a behaviour is commonly observed in the prompt emission spikes of GRBs (e.g. Ford et al. 1995), which are produced in internal shocks. Further evidence of late engine activity comes from both the flat part of the light curve $\left(\alpha_{2} \approx 0.5\right.$, see Sect. 4.3$)$ and possibly by the presence of the late-time bump observed at $t \sim 1.7 \times 10^{5} \mathrm{~s}$.

Following the discovery of a flare in the afterglow of XRF 050406, initially reported by Burrows et al. (2005b), many others were identified: GRB 050502B (Falcone et al. 2006), GRB 050724 (Barthelmy et al. 2005b) and GRB 050904 (Cusumano et al. 2005c), just to mention a few. At the time of writing (2005 Oct.), $50 \%$ of the bursts detected by XRT which were immediately re-pointed towards showed flares, making flaring quite a common behaviour. Furthermore, all the characteristics of the XRF 050406 flares have now been observed in most flaring GRBs (see Burrows et al. 2005c for a recent review). For example, highly significant spectral evolution throughout the flare has been reported in GRB 050502B (which was the brightest observed so far) and GRB 050724. In several cases the flares present large amplitudes and occur on short timescales. Furthermore, several flares are often observed in the same event, at times ranging from $\sim 100 \mathrm{~s}$ to $10^{4}-10^{5} \mathrm{~s}$ after the burst. Finally, in most cases the afterglow is clearly present before the onset of the flare, and has consistent decay slope and flux levels with after the flare. The present case shows that flares are present both in XRFs and in GRBs. Since flares are likely tied to the central engine activity, this finding further supports the idea that a similar mechanism is at work for both kind of events (Fan \& Wei 2005).

\subsection{The $X$-ray afterglow light curve}

The prompt reaction of Swift has allowed us to observe the $\mathrm{X}$-ray light curves of GRB afterglows starting from a few tens of seconds after the burst explosion. In most cases the X-ray light curves are characterized by an initial steep decay (up to $\sim 500 \mathrm{~s}$ ) followed by a shallow decay, and then by a steeper decay with a second break normally occurring at a few thousand seconds later (Chincarini et al. 2005; Nousek et al. 2006). The early steep decay seen in the X-ray light curve can be explained as the tail of the prompt emission (however, see
Panaitescu et al. 2005). The few cases where the XRT light curve lies well above the extrapolation of the prompt emission into the X-ray band can be explained either by a strong spectral evolution or by an X-ray flare with the maximum located before the XRT observation (Tagliaferri et al. 2005). There are other instances where the first steep decay is not observed at all (e.g. Campana et al. 2005).

In the case of XRF 050406, however, the initial slope is shallower than the steep values $3 \lesssim \alpha \lesssim 5$ observed in other early afterglows (Tagliaferri et al. 2005). Moreover, the curvature relation $\alpha=\beta+2$ (Kumar \& Panaitescu 2000; Dermer 2004 ) is not satisfied, even after taking into account the effects pointed out by Zhang et al. (2005) that would alter such relation. Therefore, we also investigate whether the initial decline seen in XRF 050406 is consistent with afterglow emission. Comparison of spectral indices and temporal decay slopes with theoretical relativistic fireball models (e.g. Table 2 in Zhang et al. 2005) indicates that the first decay index $\alpha_{1}=1.58 \pm 0.17$ and energy index $\beta=1.1 \pm 0.3$ rule out fast cooling models (for which the injection frequency $v_{\mathrm{m}}$ exceeds the cooling frequency $v_{\mathrm{c}}$ ) for $v<v_{\mathrm{m}}$. For $v>v_{\mathrm{m}}$, the $\alpha(\beta)=(3 \beta-1) / 2$ closure relation is satisfied within the errors and an electron power-law distribution index $p \approx 2.5$ is obtained. The same relation holds for the slow cooling regime (where $v_{\mathrm{c}}>v_{\mathrm{m}}$ ) for $v>v_{\mathrm{c}}$ (both wind and ISM). In this case a consistent solution is also found for $v_{\mathrm{m}}<v<v_{\mathrm{c}}$, although with a large $p \approx 3$. The ISM environment is favoured on the basis of a better satisfied closure relation. In conclusion, the spectral indices and temporal decay slopes of the first part of the X-ray curve can be interpreted in terms of relativistic fireball models, even though the large uncertainties associated with the slopes do not allow us to choose among the available models.

An alternative explanation for the initial XRT emission is the presence of an additional flare which started before the beginning of the XRT observation, and of which we only see the decaying part. The superposition of two (and possibly more, fainter) flares would then mimic the initial steep power law decay. However, this interpretation seems less likely since recent Swift observations of X-ray flares within the first several hundred seconds of the prompt emission all had temporal decay indices much steeper than the observed XRF 050406 pre-flare index.

At $t \sim 4400 \mathrm{~s}$ the XRT light curve breaks to $\alpha_{2} \approx 0.5$. Such a flat decay cannot be explained in terms of the standard afterglow model. The only possibility would be to observe, in the fast cooling regime, the segment with $v_{\mathrm{c}}<v<v_{m}$ (where $\alpha=0.25$ is expected, marginally consistent with the observed value). However, the fast cooling regime is expected to end much earlier. To maintain the observed decay unbroken up to $\sim 10^{6} \mathrm{~s}$, large values of the equipartition parameters $\varepsilon_{e}$ and $\varepsilon_{B}$ or of the Compton parameter would be required. We consider this possibility quite unlikely. Another possibility is that the angular energy profile of the fireball is not trivial (a structured jet), so that emission coming from the (brighter) wings of the jet may increase the observed flux as the fireball Lorentz factor decreases (Panaitescu et al. 2005).

An interesting explanation for the shallow-decay phase is injection of new energy into the fireball through refreshed 
shocks (Sari \& Mészáros 2000; Zhang \& Mészáros 2001). For this to happen, the energy release inside refreshed shocks must be sizeable, since the whole fireball dynamics has to be modified. Assuming an energy injection rate $\dot{E} \propto t^{-q}$, we find $q$ in the range 0 to 0.5 depending on the model details (Zhang et al. 2005). In this model, the initial part of the XRT afterglow light curve can be due to standard afterglow emission only if the fireball evolution is not influenced at these stages. Indeed, the energy supply provided by refreshed shocks is steadily growing, and at the beginning it cannot alter the fireball dynamics. In this case, the break would identify the time when the new, injected energy is comparable to the fireball energy. On the contrary, if the first XRT phase were due to late engine activity, then the energy injection could have begun much earlier and its emission would have been masked.

Integration of the light curve from the onset of the flat slope phase yields $\mathcal{F} \approx 3 \times 10^{-8}\left(t_{\text {end }} / 7.6 \times 10^{5} \mathrm{~s}\right)^{0.5} \mathrm{erg} \mathrm{cm}^{-2}$, where $t_{\text {end }}$ is the time at which the shallow phase ends, for which we can only set a lower limit. We note that this depends weakly on the onset time of the shallow phase, therefore the calculated fluence is correct in both presented scenarios. For comparison, the amount of energy released during the steep phase of the light curve (excluding the flare) is $\mathcal{F} \approx 2 \times$ $10^{-8}\left(t_{\text {start }} / 100 \mathrm{~s}\right)^{-0.6} \mathrm{erg} \mathrm{cm}^{-2}$. We note that the shallow phase lasts a considerable time.

Zhang et al. (2005) propose three explanations for the energy injection mechanism. In the impulsive case (Sari \& Mészáros 2000), the central engine ejects material with a wide distribution of Lorentz factors. In this case, slower moving shells will catch the fireball at a later time. We can estimate the minimum Lorentz factor as $\Gamma_{\text {min }} \lesssim 2\left(E_{\text {iso }, 50} / n_{0}\right)^{1 / 8}(1+z)^{3 / 8}$, where $E_{\text {iso }}=E_{\text {iso, } 50} \times 10^{50} \mathrm{erg}$ is the isotropic-equivalent energy, and $n_{0}$ is the external medium particle density in units of $\mathrm{cm}^{-3}$. This implies that the acceleration process works from ultra- to mildly-relativistic velocities. Within the putative Poyinting flux scenario (Zhang \& Kobayashi 2005), the energy supply is provided by the transfer of magnetic energy to the fireball, and the time at which the injection stops is related to the ratio $\sigma$ of the electromagnetic to baryonic kinetic energy. If this scenario is correct, we can infer a lower limit of $\sigma=\left(t_{\text {end }} / t_{\text {start }}\right)^{1-q}>10-100$, for $q=0.5-0$, where $t_{\text {start }}<t_{\mathrm{b}}$ is the start time of injection. Therefore, after the end of the energy transfer phase, the energy of the blast-wave would be increased by a comparable factor. In the third scenario (the prolonged energy output by the central engine, Zhang \& Mészáros 2001), the end of the injection phase is simply the end of the engine activity. In this case, this activity produces a large amount of energy, particularly so since the radiative efficiency may be lower during the late afterglow than during the prompt emission, as is generally the case. This was previously noticed by Nousek et al. (2006) in a sample of several Swift GRBs.

The monitoring of XRF 050406 was discontinued 22 days after the trigger. By then, the source was no longer detectable and only a $3-\sigma$ upper limit could be drawn at $\approx 3.6 \times$ $10^{-4}$ counts s$^{-1}$. In order for the afterglow energy not to diverge, a further, late break is necessary. One interesting possibility is that this may be due to seeing the edge of the jet. A steepening in the light curve is expected when the fireball
Lorentz factor becomes comparable to the inverse of the jet half-opening angle. Such a late break is not unexpected for an XRF. The few XRFs with known redshift (Soderberg et al. 2004; Bersier et al. 2005; Fynbo et al. 2004) have a very low isotropic-energy release, and this may be at least in part accommodated if they have very wide jets. This picture is consistent with the result found by Frail et al. (2001; see also Ghirlanda et al. 2004), who found that low-energy GRBs tend to have wider opening angles. Using the standard formalism (Rhoads 1999; Sari et al. 1999), the jet half-opening angle is $\vartheta_{\mathrm{j}}=16 t_{\mathrm{j}, 6}^{3 / 8} n_{0}^{1 / 8}(\eta / 0.2)^{1 / 8} E_{\text {iso,50 }}^{-1 / 8} \mathrm{deg}$, where $t_{\mathrm{j}}=t_{\mathrm{j}, 6} \times 10^{6} \mathrm{~s}$ is the jet break time and $\eta$ is the burst radiative efficiency. Therefore, using our lower limit on the jet break time $t_{\mathrm{j}} \gtrsim 10^{6} \mathrm{~s}$, we can infer a lower limit on the jet half-opening angle of $16 \mathrm{deg}$. This value is at the high end of the distribution of jet angles (Bloom et al. 2003b).

\section{Summary and conclusions}

$\mathrm{XRF} 050406$ is classified as an X-ray flash, with fluence $\sim 1 \times$ $10^{-7} \mathrm{erg} \mathrm{cm}^{-2}(15-350 \mathrm{keV})$, a soft spectrum $\left(\Gamma_{\gamma}=2.65\right)$, no significant flux above $\sim 50 \mathrm{keV}$ and a peak energy $E_{\mathrm{p}}<15 \mathrm{keV}$. Its main characteristics are however not qualitatively different from those of normal GRBs. As observations accumulate, it becomes clear that these two classes of phenomena share many properties, and both have afterglows with similar characteristics. This is a clue that both events may have a common origin.

XRF 050406 is the first Swift-detected burst that showed a flare in its X-ray light curve, a feature now found in $\sim 50 \%$ of the XRT afterglows. The flare peaked at $\sim 210 \mathrm{~s}$ after the BAT trigger $(\sim 61 \mathrm{~s}$ in the rest frame). The best fit of the afterglow decay is obtained with a broken power law with $\alpha_{1}=1.58 \pm 0.17, \alpha_{2}=0.50_{-0.13}^{+0.14}$, and a break at $\sim 4400 \mathrm{~s}$ after the BAT trigger. The mean photon index is $\Gamma_{\mathrm{X}}=2.1 \pm 0.3$. During the X-ray flare a flux variation of $\delta F / F_{\text {peak }} \sim 6$ in a timescale $\delta t / t_{\text {peak }} \ll 1$ is observed, and its measured fluence in the $0.2-10 \mathrm{keV}$ band is $\sim 1.4 \times 10^{-8} \mathrm{erg} \mathrm{cm}^{-2}[(2.0 \pm 1.4) \times$ $10^{50} \mathrm{erg}$, which corresponds to $1-15 \%$ of the prompt fluence. We argued that the flare-producing mechanism is late internal shocks, which implies that the central engine is still active at $t \sim 210 \mathrm{~s}$, though with a reduced power with respect to the prompt emission. We showed possible indications of spectral variations during the flare, and a flattening of the X-ray light curve after $t \sim 4400 \mathrm{~s}$ in support of continued central engine activity at late times.

Since XRF 050406 was observed, flares have been detected by XRT in both X-ray flashes and normal GRBs, indicating that flares are linked to some common properties of both kinds of bursts, and probably tied to their central engine.

Acknowledgements. This work is supported at OAB by ASI grant I/R/039/04, at Penn State by NASA contract NAS5-00136 and at the University of Leicester by PPARC. We gratefully acknowledge the contributions of dozens of members of the XRT and UVOT team at OAB, PSU, UL, GSFC, ASDC, and MSSL and our subcontractors, who helped make this instrument possible. 


\section{References}

Amati, L., Frontera, F., Tavani, M., et al. 2002, A\&A, 390, 81

Band, D., Matteson, J., Ford, L., et al. 1993, ApJ, 413, 281

Barthelmy, S. D., Barbier, L. M., Cummings, J. R., et al. 2005a, Space Sci. Rev., 120, 143

Barthelmy, S. D., Chincarini, G., Burrows, D. N., et al. 2005b, Nature, 438, 994

Berger, E., Oemler, G., \& Gladders, M. 2005a, GRB Coordinates Network, 3185

Berger, E., Kulkarni, S. R., Fox, D. B., et al. 2005b, ApJ, 634, 501

Bersier, D., Fruchter, A. S., Strolger, L.-G., et al. 2005, ApJ, submitted

Bloom, J. S., Fox, D., van Dokkum, P. G., et al. 2003a, ApJ, 599, 957

Bloom, J. S., Frail, D. A., \& Kulkarni, S. R. 2003b, ApJ, 594, 674

Burrows, D. N., Hill, J. E., Nousek, J. A., et al. 2005a, Space Sci. Rev., 120, 165

Burrows, D. N., Romano, P., Falcone, A., et al. 2005b, Science, 309, 1833

Burrows, D. N., Romano, P., Godet, O., et al. 2005c, Proc. of X-Ray Universe 2005 [arXiv: astro-ph/0511039]

Butler, N. R., Sakamoto, T., Suzuki, M., et al. 2005, ApJ, 621, 884

Campana, S., Antonelli, L. A., Chincarini, G., et al. 2005, ApJ, 625, L23

Capalbi, M., Perri, M., Romano, P., et al. 2005, GRB Coordinates Network, 3184

Cash, W. 1979, ApJ, 228, 939

Chincarini, G., Moretti, A., Romano, P., et al. 2005, ApJ, submitted [arXiv: astro-ph/0506453]

Cusumano, G., Kennea, J., Burrows, D. N., et al. 2005a, GRB Coordinates Network, 3181

Cusumano, G., Mangano, V., Angelini, L., et al. 2005b, ApJ, in press [arXiv: astro-ph/0509737]

Cusumano, G., Mangano, V., Chincarini, G., et al. 2005c, Nature, in press

De Pasquale, M., Piro, L., Gendre, B., et al. 2005, A\&A, submitted [arXiv: astro-ph/0507708]

Dermer, C. D. 2004, ApJ, 614, 284

Dickey, J. M., \& Lockman, F. J. 1990, ARA\&A, 28, 215

Falcone, A. D., Burrows, D. N., Lazzati, D., et al. 2006, ApJ, in press [arXiv: astro-ph/0512615]

Fan, Y. Z., \& Wei, D. M. 2005, MNRAS, 364, L42

Fenimore, E. 1999, ApJ, 512, 683

Fishman, G. J., \& Meegan, C. A. 1995, ARA\&A, 33, 415

Ford, L. A., Band, D. L., Matteson, J. L., et al. 1995, ApJ, 439, 307

Fox, D. 2004, GRB Coordinates Network, 2626

Frail, D. A., Kulkarni, S. R., Sari, R., et al. 2001, ApJ, 562, L55

Fynbo, J. P. U., Sollerman, J., Hjorth, J., et al. 2004, ApJ, 609, 962

Galli, A., \& Piro, L. 2005, A\&A, submitted [arXiv: astro-ph/0510852]

Gehrels, N., Chincarini, G., Giommi, P., et al. 2004, ApJ, 611, 1005

Ghirlanda, G., Ghisellini, G., \& Lazzati, D. 2004, ApJ, 616, 331

Heise, J., in't Zand, J., Kippen, R. M., \& Woods, P. M. 2001, Gammaray Bursts in the Afterglow Era, 16

Hill, J. E., Burrows, D. N., Nousek, J. A., et al. 2004, Proc. SPIE, 5165,217
Ioka, K., Kobayashi, S., \& Zhang, B. 2005, ApJ, 631, 429

King, A., O'Brien, P. T., Goad, M. R., et al. 2005, ApJ, 630, L113

Kobayashi, S., Zhang, B., Mészáros, P., \& Burrows, D. N. 2005, ApJ, submitted [arXiv: astro-ph/0506157]

Kouveliotou, C., Meegan, C. A., Fishman, G. J., et al. 1993, ApJ, 413, L101

Krimm, H., Barbier, L., Barthelmy, S., et al. 2005, GRB Circ. Network, 3183

Kumar, P., \& Panaitescu, A. 2000, ApJ, 541, L51

Lamb, D. Q., Donaghy, T. Q., \& Graziani, C. 2004, New Astron. Rev., 48,423

Landsman, W., Hunsberger, S., Breeveld, A., et al. 2005, GRB Coordinates Network, 3182

Lazzati, D., Rossi, E., Covino, S., Ghisellini, G., \& Malesani, D. 2002, A\&A, 396, L5

Levan, A., Patel, S., Kouveliotou, C., et al. 2005, ApJ, 622, 977

Moretti, A., Perri, M., Capalbi, M., et al. 2006, A\&A, 448, L9

Nakar, E., Piran, T., \& Granot, J. 2003, New Astron. Rev., 8, 485

Nousek, J. A., Kouveliotou, C., Grupe, D., et al. 2006, ApJ, in press [arXiv: astro-ph/0508332]

Panaitescu, A., Mészáros, P., Gehrels, N., Burrows, D. N., \& Nousek, J. A., 2005, MNRAS, submitted [arXiv: astro-ph/0508340]

Parsons, A., Barthelmy, S., Cummings, J., et al. 2005, GRB Coordinates Network, 3180

Perna, R., Armitage, P. J., \& Zhang, B. 2006, ApJ, 636, L29

Piran, T. 1999, Phys. Rep., 314, 575

Piro, L., Costa, E., Feroci, M., et al. 1999, ApJ, 514, L73

Piro, L., De Pasquale, M., Soffitta, P., et al. 2005, ApJ, 623, 314

Rees, M. J., \& Mészáros, P. 1998, ApJ, 496, L1

Rhoads, J. E. 1999, ApJ, 525, 737

Rol, E., Schady, P., Hunsberger, S., et al. 2005, GRB Coordinates Network, 3186

Romano, P., Cusumano, G., Campana, S., et al. 2005, Proc. SPIE, 5898,357

Roming, P. W. A., Kennedy, T. E., Mason, K. O., et al. 2005, Space Sci. Rev., 120, 95

Sakamoto, T., Lamb, D. Q., Kawai, N., et al. 2005, ApJ, 629, 311

Sari, R., \& Piran, T. 1999, ApJ, 520, 641

Sari, R., Piran, T., \& Halpern, J. P. 1999, ApJ, 519, L17

Sari, R., \& Mészáros, P. 2000, ApJ, 535, L33

Schady, P., Mason, K. O., Osbourne, J. P., et al. 2006, ApJ, in press [arXiv: astro-ph/0601182]

Soderberg, A. M., Kulkarni, S. R., Berger, E., et al. 2004, ApJ, 606, 994

Tagliaferri, G., Goad, M. R., Chincarini, G., et al. 2005, Nature, 436, 985

Vaughan, S., Goad, M. R., Beardmore, A. P., et al. 2006, ApJ, 638, 920

Zhang, B., \& Mészáros, P. 2001, ApJ, 552, L35

Zhang, B., \& Mészáros, P. 2004, Int. J. Mod. Phys. A, 19, 2385

Zhang, B., Fan, Y. Z., Dyks, J., et al. 2005, ApJ, in press [arXiv: astro-ph/0508321]

Zhang, B., \& Kobayashi, S. 2005, ApJ, 628, 315 